\title{
Graphite Intercalation Compounds
}

\section{Model Systems for Low Dimensional Phenomena}

\section{A. Magerl, Grenoble}

(Institut Laue-Langevin)

Highly anisotropic materials have received widespread attention in recent years because they can act as model systems for the study of the influence of dimensionality on physical properties. Two-dimensional (2D) systems have been approximated in such structures as free standing films, electrons on liquid helium, gases adsorbed on surfaces, and molecules intercalated in layered host materials. In this latter group, graphite-based compounds have been of particular interest in view of the anisotropy of the host and the structural simplicity of the layers. Within the planes, the carbon-carbon distance is only $1.42 \AA$ and the atoms are bound by one the strongest covalent bonds known, whereas the interplane bonding is of much weaker van der Waals type with a layer separation of $3.35 \AA$. This weak bond can open up easily and a large variety of molecules, ranging from simple donors like the alkali metals to acceptors like the halides and including even large organic molecules, can be intercalated.

One of the most fascinating properties of graphite intercalation compounds (GICs) is their unique ability to form stages. A stage $n$ means that only every $n^{\text {th }}$ van der Waals gap is filled with the intercalant in a regular long ranged fashion. In a stage 1 compound for example, every interlayer gap is intercalated and in a stage 3 compound there are three successive graphite planes followed by an intercalated layer (see Fig. 1). The in-plane concentration of the intercalate depends only weakly on the

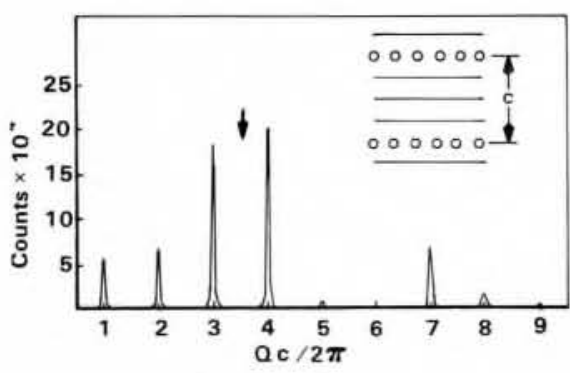

Fig. $1-(00 \%)$ difraction scan on stage 3 $C_{36} C s$ with $c=19.1 \AA$. The arrow marks the position of the first Bragg peak for graphite. The insert shows the arrangement of the layers for a stage 3 compound with - for graphite layers and ooo for intercalated layers (see Ref. 2). stage number except for some stage 1 compounds. Advantage can be taken of the ability of GICs to form stages to study dimensional crossover effects; e.g. 3D crystallographic and magnetic structures are found in low stage compounds at low temperatures, whereas at high temperatures and/or in high stage compounds, 2D ordering is observed. At sufficiently high temperature, the configurational entropy will dominate over other interactions, leading to stage 1 compounds which could have a small concentration of the intercalate. Although this has been predicted theoretically, there are only a few experimental indications of dilute stage 1 phases in an accessible temperature range.

The ability of graphite-based compounds to form stages is to be contrasted with other layered materials like clays or metal dichalcogenides which, in general, exist only in stage 1 . However, for these compounds the in-plane concentration of the intercalated species can be varied over a wide range.

GICs constitute a very large family within the group of layered materials. A vast variety of molecules can be intercalated in various stages and literally many hundreds of compounds have been synthesized and characterized. Moreover, the host material itself can be obtained in such different forms as graphite fibres, 1D ordered pyrolytic gra phite and 3D ordered single crystals. The variety of chemical and physical properties of GICs explains the present high interest in both basic research, where GICs serve as a playground for scientists in which they can find and study intriguing new phenomena, and in application-oriented research, where GICs can be tailored to meet specific requirements.

In this article, many exciting features of GICs will have to be omitted such as their electronic structure or electric conductivity which can approach values as low as for an insulator in the direction of the c-axis, whereas an in-plane conductivity similar to that of $\mathrm{Cu}$ at room temperature has been reported. A few recent publications are given in Ref. 1 for the reader further interested in the subject. In the following, I will briefly describe some aspects of the synthesis of GICs and then will focus on crystallogra- phic structures and phase transitions, on molecular diffusion, and on magnetism.

\section{Synthesis}

Compounds can be prepared in many different ways. Intercalation from the gas phase in a two-zone furnace, where the temperature of the graphite and of the intercalant is controlled independently, is a frequently applied technique, in particular, in cases of simple mole cules like the alkali metals. Electrolytic charging has been applied very successfully to many substances such as acids. Both these techniques are well suited for the production of well defined compounds of high stage purity. Other techniques, such as the immersion of graphite in a solution or in a liquid like molten Li can also be used. Increasing emphasis has recently been put on the study of ternary compounds, in which two different types of molecule are introduced. These can be prepared from "normal" GICs in a second intercalation process: e.g. alkali-GICs exposed to a few torr of hydrogen atmosphere at low temperature will readily absorb the gas in the layers already containing the metal. A modified version of this procedure leads to mixed ternary compounds like stage $1 \mathrm{C}_{8} \mathrm{~K}_{x} \mathrm{Rb}_{1-x}$ where $\mathrm{Rb}$ can be substituted progressively by $\mathrm{K}$. Many GICs are unstable in air. However they can be stored in inert gas or under vacuum for a long time without signs of deterioration.

The purity of the stage of a sample can be checked by (OOl) diffraction scans from which the distance between intercalated layers can be determined. $X$-rays are an appropriate probe for small samples, whereas neutron diffraction with its larger penetration depth is better for large samples. The neutron diffraction pattern shown in Fig. 1 which was taken on a Cs-GIC of $30 \mathrm{~cm}^{3}$ reveals only Bragg reflections corresponding to a repeat distance of $12.1 \AA$. This demonstrates that this unusually large sample is pure stage 3 .

\section{Crystallographic Structures}

As a result of possible stacking sequences of the layers, the true crystallographic repeat distance in the c-direction can be considerably longer than in 
a

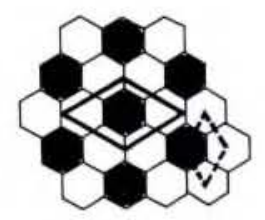

b
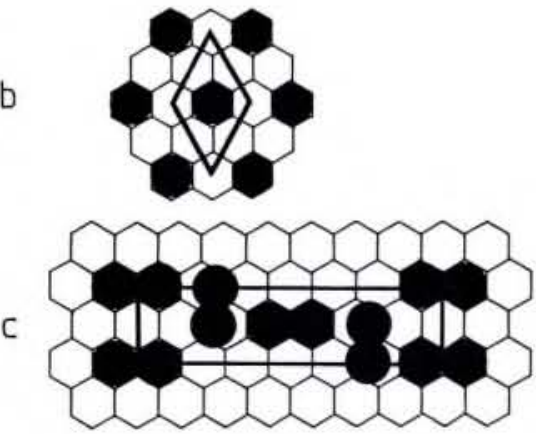



Fig. 2 - Examples of in-plane ordering of the intercalants in GICs. The carbon atoms are located at the intersections of the honeycomb network and the position of the intercalant atoms projected on to a graphite plane are indicated by the spots. The size of the intercalants is not drawn to scale. Part a shows the $(\sqrt{ } 3+\sqrt{ } 3)$ structure (e.g. LiC 6$)$. The graphite unit cell in the basal plane is given by the dashed lines. Part b gives the $(2 \times 2)$ structure as found for stage 1 heavy alkali compounds (e.g. $\left.C_{g} K\right)$. The $(\sqrt{ } 3 \times 7)$ structure as found for stage $4 C_{28}$ $\mathrm{Br}_{2}$ is shown in part c. Part d gives the low-temperature ordering of stage 2 alkali GICs. Patches of intercalants occupy hexagon centers in an "almost" commensurate fashion based on a $(\sqrt{ } 7 \times \sqrt{ } 7)$ structure. These patches are separated by a network of honevcomb-shaped discommensurations.

dicated by the stage number $n$. The inplane unit cell of the stage 1 heavy alkali $(\mathrm{K}, \mathrm{Rb}, \mathrm{Cs})$ compounds has a $(2 \times 2)$ structure (Fig. 2b): the basis vectors in real space are twice the length of the basis vectors for graphite. The cell contains eight carbons and covers four equivalent graphite hexagons. For $\mathrm{K}$ and $\mathrm{Rb}$ the alkali occupy these sites in a commensurate fashion, and the complete stacking sequence is $A \alpha A \beta A \gamma A \delta$ with capital letters and Greek letters referring to graphite and alkali layers, respectively. This yields a c-axis of $21.4 \AA$ and $22.6 \AA$ for $C_{8} K$ and $C_{8} R b$, respectively. $C_{8} C s$ has a different $A \alpha A \beta A \gamma$ stacking sequence and the repeat distance is 17.8 $\AA$. The in-plane unit cell for stage $1 \mathrm{Lihas}$ a $(\sqrt{3} \times \sqrt{3})$ structure which accommodates six carbon and three graphite hexagons (Fig. 2a). However, the stacking sequence is simple $A \alpha$, which gives $\mathrm{LiC}_{6}$ the shortest $\mathrm{c}$-axis of $3.71 \AA$ of all GICs. At high temperature, the 3D ordered intercalated layers of stage 1 compounds show an order-disorder transition. For stage $1 \mathrm{Li}$ or sub-stoichiometric stage $1 \mathrm{Cs}$, the alkali melts into a lattice-gas type structure where on the average all hexagon centres are occupied by alkali with equal probability. For stoichiometric $\mathrm{C}_{8} \mathrm{Cs}$, the alkali seems to form a $2 \mathrm{D}$ liquid which is incommensurate with the graphite substrate.

Stages 2 and higher reveal already at room temperature in-plane liquid-like diffuse scattering from the alkali, without showing interlayer correlations along least locally as a lattice gas. Measure ments on single crystals show an angu- lar variation of the diffuse intensity which has the hexagonal symmetry of the graphite. This modulation becomes more pronounced at lower temperatures and demonstrates strong orientational correlations between the disordered alkali and the substrate.

At sufficiently low temperatures, the diffuse peaks transform into sharp diffraction spots and a rich pattern of satellite peaks shows up. At the same time 3D correlations develop, in which the alkali have ordered in a hexagonal structure that is both incommensurate with the graphite lattice and rotated with respect to it. The discussion of the complex diffraction patterns at low temperature has led to controversial interpretations. Recently a model involving a static distortion wave within the alkali layers has attracted great interest. In this model, it is assumed that the alkali forms patches in which the atoms occupy hexagon centres in an "almost" commensurate fashion based on a $(\sqrt{ } 7 \times \sqrt{ } 7)$ structure rotated against the graphite lattice by $19.1^{\circ}$ (Fig. 2d). These islands, with linear dimensions of say 10 times the length of the graphite basis vector, are separated from each other by a network of honeycomb-shaped incommensurable lines.

Alkali-based GICs might be considered as structurally simple compounds. However, the fine balance between competing interactions such as electronic and elastic forces both in-plane and the c-axis. Although this disordered state has been studied extensively, there is no generally accepted picture as to liquid, where the positions of the alkali are uncorrelated with the substrate, or to

\section{THE GLENN BROWN AWARD}

\section{THE GLENN BROWN AWARD FOR OUTSTANDING PhD THESIS IN THE FIELD OF LIQUID CRYSTALS}

The Planning and Steering Committee of the International Liquid Crystal Conferences has instituted a prize in honour of Professor Glenn Brown, for an outstanding PhD Thesis in the field of liquid crystal research. The prize will be awarded at each of the forthcoming biennial conferences, beginning with the 11th International Liquid Crystal Conference in Berkeley, June 29 to July 4, 1986.

The Award Committee consists of S. Chandrasekhar, Bangalore, $\mathbf{S}$. Kobayashi, Tokyo, H. Sackmann, Halle, and S.T. Lagerwall, Göteborg (chairman). Nominations are now solicited and should be sent to the chairman before December 15, 1985. There are no restrictions regarding the subfield, thus theoretical, experimental or applied work is equally welcome on both thermotropic and lyotropic systems. A thesis can be nominated by anyone and should not be more than three years old.

The nominating person should state his name and affiliation as well as those of student and thesis adviser. The nomination should be accompanied by a detailed abstract (about three pages) in English A first selection will take place on the basis of the delivered abstracts and a limited number of candidates will then be asked to send in the complete thesis. Correspondence should be sent to

Prof. S.T. Lagerwall. Physics Department

Chalmers University of Technology, S - 41296 Göteborg. Sweden 
out-of-plane results in an unexpected manifold of atomic order, and measurements on single crystals have been required to unravel the details of the structures. GICs formed from multi-atomic in tercalants can be expected to exhibit an even richer variety of ordering phenomena, due to the molecular orientation which provides additional degrees of freedom. The structures and phase transitions have been studied particularly well for $\mathrm{Br}_{2}$ and $\mathrm{HNO}_{3}$ GICs.

In stage $4 \mathrm{C}_{28} \mathrm{Br}_{2}$ at room temperature there are three rotationally distinct sublattices. The in-plane unit cell is rectangular with a $(\sqrt{ } 3 \times 7)$ structure (Fig. $2 c)$ with the long axis pointing along a graphite [110] direction. The cell contains four $\mathrm{Br}_{2}$ molecules which are commensurate with the host lattice. At $342.2 \mathrm{~K}$ a solid-solid phase transition occurs, in which only the long axis becomes incommensurate with the substrate, while the short axis remains in register. This so-called stripe domain phase is analogous to the discommensuration model proposed for the low temperature ordering of high stage alkali GICs discussed earlier. However for $\mathrm{C}_{28} \mathrm{Br}_{2}$ the domain walls form only a $1 \mathrm{D}$ grid instead of a $2 \mathrm{D}$ network due to the molecular structure of the intercalate. The origin of the unusual 1D incommensurate phase is to be found in the difference in the thermal expansion between the rigid host and the soft and anisotropic $\mathrm{Br}_{2}$ layers. At high temperature the mismatch becomes sufficient for the creation of domain walls in the direction of the long axis of the unit cell.

The in-plane ordering for high stage $\mathrm{HNO}_{3}$ shows a similar pattern. At low temperatures, a large supercell is formed which is probably commensurate with the graphite. Above $210 \mathrm{~K}$ the lattice becomes again incommensurate in one direction only and above $250 \mathrm{~K}$ an anisotropic disordered state develops which is orientationally correlated with the structure of the graphite substrate. A first determination of the molecular orientation indicated that the planar $\mathrm{HNO}_{3}$ molecules form a double layer in the van der Waals gap, whereas more recent data provide evidence for a nearly perpendicular orientation of the $\mathrm{HNO}_{3}$ molecule in between the graphite planes.

\section{Diffusion}

The diffusion of intercalates has been studied because of both the intrinsic interest in $2 \mathrm{D}$ particle dynamics and the relation to the rapid intercalation process. In addition, valuable information on topics such as structures and phase transitions can be obtained in favourable

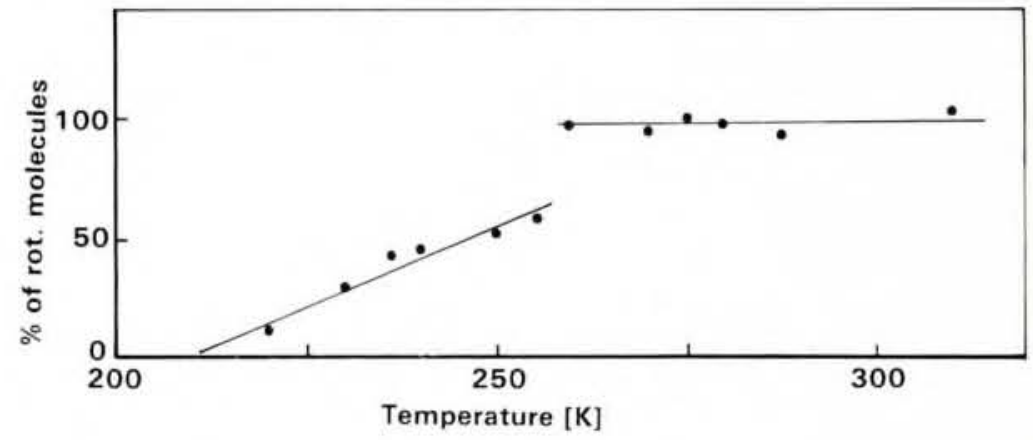

Fig. 3 - Fraction of molecules participating in the in-plane rotation for a stage $2 \mathrm{HNO}_{3} \mathrm{GIC}$ (Ref. 3).

cases. Particle diffusion leads to small energy transfers centred around zero energy transfer which can be observed in a neutron scattering experiment. Detailed information on the microscopic diffusion mechanism can be obtained from the wavevector dependence of this so-called quasi-elastic line broadening.

There has been some question as to whether the disordered high temperature phases correspond to a liquid or to an amorphous state. The spatial correlations between the molecules are similar in both cases, and therefore cannot be distinguished from a diffraction pattern. The main difference lies in the particle mobility, which is very small for an amorphous body, but large for a liquid.

In-plane diffusion constants of order $10^{-5} \mathrm{~cm}^{2} \mathrm{~s}^{-1}$ which are similar to the diffusion constants for a "normal" 3D liquid have been observed for stage 2 alkali GICs at room temperature. Therefore an assignment of the disordered phases to a 2D liquid phase is justified. The quasi-elastic linewidth has been found to oscillate around a mean value for wavevectors $Q$ larger than $1 \AA^{-1}$. This demonstrates that preferred jump vectors which seem to be related to the graphite substrate exist for the alkali. Such a behaviour strongly suggests latticegas type correlations for the disordered alkali layers.

Because of the overwhelmingly large neutron-proton cross-section, the diffusion of a molecule like $\mathrm{HNO}_{3}$ can be monitored by the motion of the hydrogen. The in-plane diffusion for this system shows different characteristic properties for each crystallographic phase. In the high temperature disordered state, both rotational and translational motions are observed. In analogy to the alkali, it is found that the translational component is best described by a jump diffusion with a displacement vector of about $2.5 \AA$. This demonstrates the decisive influence of the graphite on the intercalate structure in the high temperature phases, in agreement with the anisotropic diffuse $X$-ray scattering pattern from single crystals. Translational motion is no longer observed in the $1 D$ stripe domain phase below $250 \mathrm{~K}$, whereas rotation of the molecule continues. Nevertheless, the phase boundary is clearly marked by a sudden drop of the rotational diffusion constant. Surprisingly, the number of molecules participating in the rotation decreases continuously until all molecules are frozen in at $210 \mathrm{~K}$, where the commensurate phase is reached (Fig. 3). This unusual feature indicates that only the molecules next to a domain wall are mobile. With decreasing temperature the number of lattice defects decreases, which is reflected by a progressively reduced quasi-elastic intensity.

\section{Lattice Dynamics}

The inelastic spectrum reflects the forces within the intercalated layers, the changes of the forces in the graphite planes and the interactions between the host and the intercalates. This provides valuable information for a large variety of chemical and physical properties. The lattice dynamics of graphite and GICs extends to very high phonon energies of $200 \mathrm{meV}$ and, in consequence only a small part of the phonon dispersion has been measured by Raman scattering and neutron spectroscopy for some selected compounds. Still these few measurements have revealed two basic properties of the phonon dispersion of GICs: First, the dominant modifications of the phonon branches of GICs with respect to graphite can be understood from the increased volumes of the crystallographic unit cells (see Fig. 2) which leads to zone folding effects, and second, intercalation only influences the forces in the immediate neighbourhood of the inserted layer. Even the changes of the force constants within the graphite planes next to the intercalated layer are only in the percent range and the strong graphitic C-C bond is maintained. 


\section{Magnetism}

Molecules with magnetic moments inserted in graphite will exhibit magnetic ordering, although the transition temperatures are lower than in the crystalline state of the intercalant. The particular interest in magnetic GICs arises from the pronounced anisotropy of interlayer to intralayer coupling which can vary by several orders of magnitude depending on the stage of the compound. Examples studied to date include $\mathrm{FeCl}_{3}$, $\mathrm{CoCl}_{2}, \mathrm{NiCl}_{2}$ and $\mathrm{MoCl}_{2}$. Also rare earth metals such as stage $1 \mathrm{EuC}_{6}$ have been synthesised and magnetic ordering has been found below $40 \mathrm{~K}$. However no higher stages have been reported for Eu. For stages $n \geq 2$ the compounds show two magnetic transitions at the temperatures $T_{4}$ and $T_{p}$. The higher temperature $T_{u}$ is independent of stage and it is assigned to a magnetic in-plane ordering, whereas the lower temperature $T_{p}$ decreases with increasing stage number and is related to a 3D magnetic ordering. The dimensionality of the magnetic ordering yields characteristic lineshapes in a neutron diffraction pattern: 3D order measured on a powder sample yields a symmetric and narrow diffraction peak whereas 2D order results in an asymmetric broad peak. This is illustrated in Fig. 4 for stage 1 and stage $2 \mathrm{FeCl}_{3}$ at $1.5 \mathrm{~K}$.

The magnetic structure has been determined for a few compounds only. $\mathrm{FeCl}_{3} \mathrm{GIC}$ is a Heisenberg system with the magnetic moments pointing along the c-axis. The in-plane spin structure is identical for 2D and 3D ordering, whereas the correlations along the $\mathrm{c}$ -
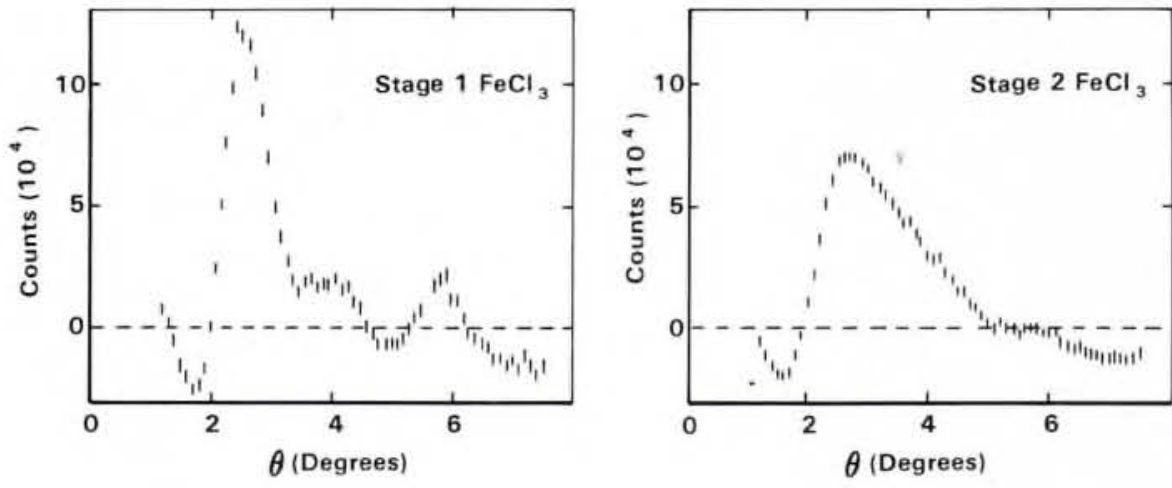

Fig. 4 - Diffraction profiles for $3 D$ and $2 \mathrm{D}$ magnetic ordering in stage 1 and stage $2 \mathrm{FeCl}_{3}$ at $1.5 \mathrm{~K}$. Measurements performed on a powder sample (Ref. 4 ).

axis in the 3D ordered spin state are not known to date. The magnetic moments for the other metal chlorides are parallel to the planes and the interlayer and intralayer interactions are antiferromagnetic and ferromagnetic, respectively. For temperatures $T_{p}<T<T_{u}$ the magnetic structure closely resembles a $2 D X Y$ phase and small deviations from infinite susceptibility and zero spontaneous magnetization as predicted by Kosterlitz and Thouless are attributed to a remnant $3 \mathrm{D}$ coupling and to the defect structure of the intercalated layer.

\section{Outlook}

Many aspects of the intercalation of graphite as presented above are also applicable to other materials like the linear chain polymer polyacetylene which can be doped in similar ways and which has recently gained widespread attention, in particular with respect to possible applications as a conductor. For the future it can be expected that the strong in- terest from both basic research and industry in the study of the physical and chemical properties of low-dimensional host materials and their intercalates will result in new synthetic compounds with novel and practical properties.

\section{REFERENCES}

1. Physics and Chemistry of Materials with Layered Structures, Vol. 6, ed. F. Levy (Reidel, Dordrecht) 1979; Proceedings of the International Conference on the Physics of In tercalation Compounds, Springer Series in Solid State Sciences, Vol. 38, ed. L. Pietronero and E. Tosatti (Springer Verlag, Berlin) 1981 ; Dresselhaus M.S. and Dresselhaus G., Light Scattering in Solids III, Topics in Applied Physics, Vol. 51, eds. M. Cardona and G. Guntherodt, pp. 3-57.

2. Zabel H. and Magerl A., Phys. Rev. B 25 (1982) 2463.

3. Batallan F., Rosenman I., Magerl A. and Fuzelier H., Phys. Rev. B, Rapid Comm. 32 (1985) 4810.

4. Simon Ch., Batallan F., Rosenman I., Schweitzer J., Lauter $\mathrm{H}$. and Vangelisti R., J. Physique 44 (1983) L-641.

\section{New Members of the European Physical Society}

\section{CATEGORY 4a)}

A. Jabali, Esfahan, Iran

K. Ghafoori-Tabrizi, Tehran, Iran

S. Narison, Montpellier, $F$

C. Sibilia, Rome, I

\section{CATEGORY 4c)}

Austrian Physical Society

W. Majerotto, Vienna

Belgian Physical Society P. De Smedt, Schilde

M. Goossens, Keerbergen

\section{French Physical Society}

J.-C. Beaufils, Villeneuve d'Asq

A. Ermolieff, Eybens

M. Lombardi, St. Martin-d'Hères M. Nusimovici, Rennes

Tran Minh Nguyet, Sucy-en-Brie A.F. de Sà Santoro,

Rio de Janeiro, Brazil
German Physical Society

C. Bennhold, Athens, OH, USA

J. Drees, Wuppertal

J. Fink, Karlsruhe

H. Hotop, Kaiserslautern

T. Peterreins, Munich

F.-K. Thielemann, Urbana, IL. USA

Netherlands' Physical Society

M. Eligh, Utrecht

Polish Physical Society

M. Koralewski, Poznan

P. Zielinski, Krakow

Portuguese Physical Society A. Barroso, Lisbon

Romanian National Committee for Physics

M. Agop, lasy

G. Zet, lasy
Spanish Royal Society

of Physics

J.-M. Lopez, Valladolid

J.L. Sanchez-Gomez, Madrid

Swedish Physical Society

A. Flodström, Lund

P. Grafström, St. Genis-Pouilly, F

H.I. Starnberg, Cambridge, UK

U. von Barth, Lund

Swiss Physical Society

A. Turnbull, Lausanne

\section{CATEGORY 4d)}

American Physical Society

P. Carruthers, Los Alamos, NM

P. Nath, Boston, MA

C. Rhodes, Chicago, IL

M. Socolovsky, Munich, D

R. Stefanski, Batavia, IL

$H$. Weerts, East-Lansing, MI
French Optical Society

H. Arditty, Marly-le-Roi

Y. Bourbin, Orsay

P. Bozec, Creteil I'Echat

C. Brehm, Marcoussis

P. Crosnier, Marly-le-Roi

J. Decombe, Poitiers

F. Desvignes, Limeil Brévannes

D. Fayoux, Les Ulis

M. Gaillard, Marcoussis

J.-P. D'Haenens, Bois d'Arcy

D. Henry, Dourdan

S. Huard, Orsay

J.-P. Huignard, Orsay

L. Jeunhomme, Marcoussis

M. Le Luyer, Evry

M. Lièvre, Orsay

A. Masson, Saint Héand

R. Miller, Paris

G. Nomarksi, Orsay

J.-P. Reboul, Saint Egrève

$J$. Robieux, Marcoussis

M. Spajer, Besançon 